\title{
Anatomía arterial de los colgajos musculares de extensor carpi radialis longus y extensor carpi radialis brevis para su uso en transferencia muscular funcional libre Arterial anatomy of the extensor carpi radialis Iongus and extensor carpi radialis brevis muscle flaps related to its use in free functioning muscle transfer
}

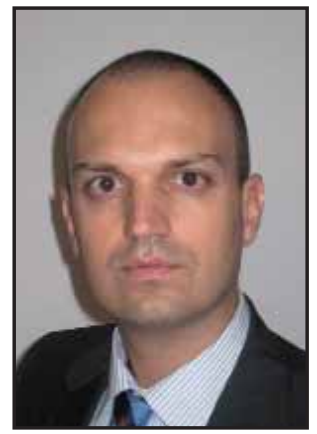

Rodríguez Lorenzo, A.

\author{
Rodríguez Lorenzo, A.*, Morris, S.F.**, Carrera Burgaya, A.***, Martelo Villar, F.*t*k
}

\section{Resumen}

El objetivo de este trabajo es documentar el aporte arterial y el patrón vascular intramuscular de los músculos Extensor Carpi Radialis Longus (ECRL) y Extensor Carpi Radialis Brevis (ECRB) para analizar su utilización como colgajos libres en transferencia muscular funcional para reanimación facial. Realizamos un estudio anatómico en 29 brazos humanos en fresco. Las técnicas de inyección utilizadas fueron la modificada de oxido de plomo y gelatina en 11 cadáveres y la de inyección pulsátil de látex color en 18. Disecamos los músculos ECRL y ECRB y sus pedículos, los fotodocumentamos y radiografiamos valorando los resultados en función del patrón vascular intramuscular, relaciones anatómicas, calibres y longitud de pedículos. Encontramos dos patrones vasculares diferentes en las 29 disecciones siguiendo la clasificación de Mathes y Nahai de la anatomía vascular de los músculos (en función del número de pedículos vasculares y su dominancia): Tipo I( $37,9 \%$ ECRL y $20,7 \%$ ECRB) y Tipo II $(62,1 \%$ del ECRL y 79,3\% del ECRB). El pedículo principal del ECRL (de diámetro medio $1,73 \mathrm{~mm}$ y longitud de pedículo media de $32,32 \mathrm{~mm}$ ) es en el $100 \%$ de los casos rama de la arteria recurrente radial y el pedículo principal del ECRB (de diámetro medio 1,11 mm y longitud de pedículo media de $27,77 \mathrm{~mm}$ ) es rama de la arteria radial en el $68,9 \%$ de los casos y de la arteria recurrente radial en el $31,1 \%$ de los casos. Concluimos que El ECRL y ECRB presentan dos tipos de patrones vasculares: tipo I y tipo II, siendo más frecuente en nuestro trabajo el tipo II, que hacen que ambos puedan ser transferidos como colgajos libres por su pedículo principal. Ambos músculos presentan un tamaño, contorno, contenido fascial importante para el anclaje de suturas y una longitud de pedículo y calibre vascular adecuados para su transferencia microvascular libre en reanimación facial. De los dos, el más realizable como colgajo libre es el ECRB ya que la relación anatómica del nervio radial con el pedículo del ECRL hace que no sea posible su transferencia como colgajo libre sin sacrificar la rama motora del nervio radial en la mayor parte de los casos.

Palabras clave Reanimación facial. Transferencia muscular funcional libre. Extensor Carpi Radialis Longus. Extensor Carpi Radialis Brevis.

Código numérico 15823-158332
Abstract

The arterial anatomy of the Extensor Carpi Radialis Longus and Brevis (ECRL, ECRB) flaps is herein described in order to provide the vascular basis to be used as free muscle transfer for facial reanimation. We used 29 fresh above-elbow human arms injected by means of two diferent techniques.Latex-injection was performed in 18 arms and the modified lead oxide-gelatine injection technique was performed in 11 arms. The ECRL and ECRB with their pedicles were dissected, photodocumented and radiographied.The number, length and calibers of the muscle pedicles were recorded. The intramuscular vascular pattern and the relations of the main pedicles of the muscles with the radial nerve were also noted. Two vascular patterns were found following the Mathes and Nahai Classification of the Vascular Anatomy of the Muscles (number of pedicles and its dominance): Type I (37,9\% of ECRL and 20,7\% of ECRB dissections) and Type II ( $62,1 \%$ of ECRL and $79,3 \%$ of ECRB dissections). The dominant pedicle for the ECRL (with an average caliber of $1,73 \mathrm{~mm}$ and an average length of pedicle of $32,32 \mathrm{~mm}$ ) is a branch of the radial recurrent artery in $100 \%$ of the dissections and the dominant pedicle for the ECRB (with an average caliber of $1,11 \mathrm{~mm}$ and an average length of pedicle of $27,77 \mathrm{~mm}$ ) is a branch of the radial artery in $68,9 \%$ of the dissections and a branch of the radial recurrent artery in $31,1 \%$ of the cases. As a conclusion, ECRL and ECRB muscle flaps present two types of vascular patterns: type I and type II.Type II pattern is the most common in our study. The anatomical features of both muscles (vascular basis,presence of an important fascial layer, contour and length of the muscle,) make them reliable as free muscles flaps for facial reanimation, however, the relation between the dominant pedicle for the ECRL muscle flap with the posterior interosseous branch of the radial nerve make not possible to free the flap without sacrificing the nerve in most of the cases.

Key words Facial reanimation. Free functioning muscle transfer. Extensor Carpi Radialis Longus. Extensor Carpi Radialis Brevis.

Numeral Gode 15823-158332

\footnotetext{
* Médico Interno Residente. Servicio de Cirugía Plástica y Quemados, complejo Hospitalario Universitario J uan Canalejo, A Coruña, España. Clinical Fellow, Canniesburn Plastic Surgery Unit, Glasgow Royal Infirmary Hospital, Reino Unido.

* Profesor Departamento de Cirugía, Anatomía y Neurobiología, Dalhausie University, Halifax, Canadá.

* Profesora Departamento de Anatomía y Embriología Humana, Universidad de Barcelona, Barcelona, España.

* J efe de Servicio de Cirugía Plástica y Quemados, Complejo Hospitalario J uan Canalejo, A Coruña, España.
}

Trabajo galardonado con el Premio Mentor de Residentes en el XLII Congreso de la Sociedad Española de Cirugía Plástica, Estética y Reparadora (SECPRE) en mayo de 2007, Valencia (España). 


\section{Introducción}

El objetivo último de la reanimación del tercio medio e inferior de la cara paralizada es lograr una sonrisa natural, simétrica y espontánea, lo que se consigue habitualmente mediante la utilización de transferencia muscular funcional libre en uno o dos tiempos quirúrgicos.

Se han empelado diferentes músculos en la transferencia muscular libre funcional, siendo los más habituales el Gracillis, Latissimus dorsi, Serrato anterior y Pectoral menor entre otros. En la mayor parte de los casos es necesaria la división del músculo en segmentos musculares para disminuir la masa muscular transferida y mejorar el contorno facial; sin embargo, durante la segmentación del músculo pueden lesionarse los vasos y nervios intramusculares.

Los músculos Extensor Carpi Radialis Longus (ECRL) y Brevis (ECRB) pertenecen al compartimento lateral del antebrazo, y su función es extender y abducir la muñeca. Ambos músculos han sido usados en Cirugía Reconstructiva, principalmente en transferencia tendinosa para tratamiento de la parálisis de los nervios mediano y cubital $(1,2)$. El valor potencial del ECRL y ECRB en reanimación facial es que son músculos que no necesitan ser acortados al transferirlos a la cara. Ambos presentan un contorno fino y un tamaño adecuado para ser utilizados en reanimación facial, pero sólo el ECRB ha sido utilizado previamente como colgajo libre (3).

Es esencial el conocimiento del aporte vascular de un colgajo para planear un procedimiento reconstructivo. Con frecuencia este conocimiento se basa en actualizar el trabajo de anatomistas del pasado mediante la aplicación de nuevas técnicas de inyección que permiten una mejor documentación de los colgajos.

El objetivo de este trabajo es documentar el aporte arterial y el patrón vascular intramuscular de los músculos ECRL y ECRB, para valorar su utilización como colgajos libres en transferencia muscular funcional para reanimación facial.

\section{Material y método}

Examinamos en fresco 29 brazos y realizamos un estudio del aporte arterial y del patrón vascular intramuscular de los músculos ECRL y ECRB según la clasificación de Mathes y Nahai (4), desde su inserción ósea proximal (cresta supracondílea lateral y epicóndilo lateral respectivamente) hasta la unión musculotendinosa.

\section{Técnicas de Inyección}

\section{Inyección óxido de plomo y gelatina}

A través de una arteria braquial inyectamos intrar- terialmente 11 brazos en fresco, usando la técnica de inyección modificada de óxido de plomo en el departamento de Anatomía, Cirugía y Neurobiología de la Universidad de Dalhausie (5) (Halifax, Canadá).Cada cadáver fresco fue inyectado con óxido de plomo, gelatina y agua a razón de $200 \mathrm{ml} / \mathrm{kg}$, y posteriormente enfriado a $4^{\circ} \mathrm{C}$ durante 24 horas antes de la disección.

\section{Inyección de látex color}

Otros 18 brazos en fresco recibieron una inyección pulsátil de látex color (1 rojo y 17 negro) a través de la arteria braquial en las instalaciones del Departamento de Anatomía y Embriología Humana de la Universidad de Barcelona (Barcelona, España). Todos los brazos recibieron una inyección intrarterial de aproximadamente $50 \mathrm{ml}$ de solución de látex introducido mediante presión manual.

\section{Disección anatómica y mediciones}

Las disecciones anatómicas fueron realizadas en las instalaciones del Departamento de Anatomía, Cirugía y Neurobiología de la Universidad de Dalhausie y del Departamento de Anatomía y Embriología Humana de la Universidad Central de Barcelona.

Todas las disecciones se hicieron bajo magnificación óptica (Keeler Loupes ${ }^{\circledR}, 3.0$ X). Resecamos la piel llegando hasta la fascia antebraquial a través de un abordaje anterior del codo, desde el tercio distal del brazo hasta la muñeca. Tras localizar el compartimento muscular lateral de antebrazo, iniciamos la disección de distal a proximal, separando el músculo Braquirradialis hasta localizar la arteria radial; ésta es seguida proximalmente hasta la bifurcación de la arteria braquial, donde se localizan la mayoría de los vasos nutricios del ECRL y ECRB.

Las disecciones fueron fotodocumentadas y se realizaron mediciones del origen, diámetro y longitud de los pedículos vasculares del ECRL y ECRB, de la distancia entre ambos, y de la arteria origen de la arteria recurrente radial, realizando las mediciones en $\mathrm{mm}$ con un calibrador (Vernier Calipers ${ }^{\circledR}$, $150 \times 0,05)$

En los 11 cadáveres inyectados con óxido de plomo y gelatina, todo el compartimento lateral del antebrazo fue disecado y extraído en bloque incluyendo la porción distal de la arteria braquial, la arteria radial y la rama sensitiva del nervio radial; se aislamos los músculos ECRL y ECRB con sus pedículos vasculares y realizamos radiografías de ambos músculos juntos y separados para determinar su patrón vascular intramuscular. 


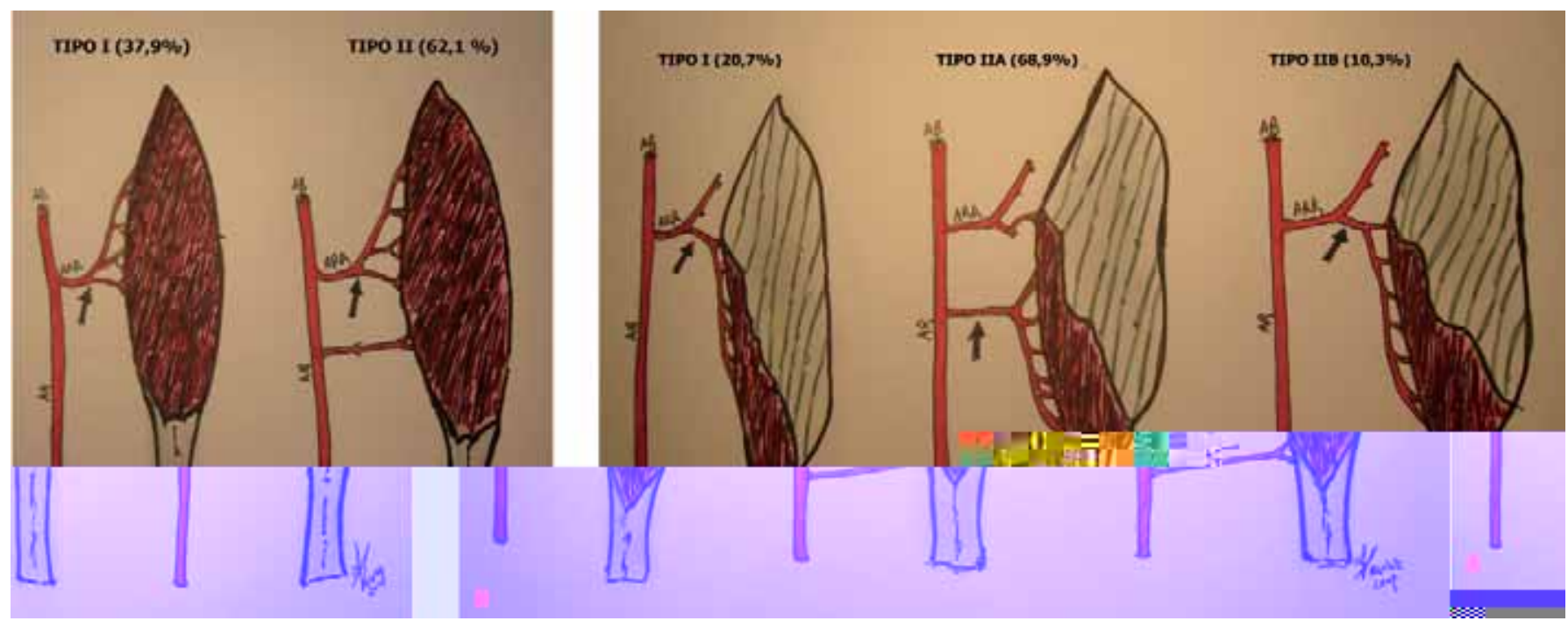

Fig. 1. Patrones vasculares. A) ECRL. Se encontraron dos patrones vasculares, el Tipo I donde el pedículo dominante (flechas negras) proviene de la ARR y el Tipo II donde además se constató la presencia de un pedículo menor proveniente de la AR. B) ECRB. Se encontraron tres tipos de patrones vasculares: Tipo I, donde el pedículo dominante sale de la ARR, Tipo IIA que presenta un pedículo dominante rama de la AR y uno o varios menores ramas de la ARR y de la AR y Tipo IIB en el que el pedículo principal sale de la ARR y presenta un pedículo menor distal rama de la AR. (AB: arteria braquial; AR: arteria radial; ARR: arteria recurrente radial).

\section{Resultados}

Encontramos 2 patrones vasculares diferentes en los 29 ECRL y ECRB disecados (Fig.1), siguiendo la clasificación de Mathes y Nahai (5) de la anatomía vascular de los músculos (en función del número de pedículos vasculares y su dominancia): Tipo I y Tipo II, es decir la vascularización es a expensas de un único pedículo vascular o un pedículo dominante y uno o varios pedículos menores, respectivamente. En todos los casos las ramas arteriales musculares circulan transversalmente dentro del músculo, por lo que el diseño de un colgajo debe evitar la sección longitudinal del músculo.

Radiográficamente, se constató en los arteriogramas la existencia de múltiples anastomosis vasculares entre el pedículo principal y los menores en ambos músculos. La medida de calibres y longitudes de pedículo se expresan en la Tabla I.

\section{Extensor Carpi Radialis Longus}

Objetivamos 3 fuentes de aporte arterial a la porción muscular del ECRL: la arteria recurrente radial(ARR), la arteria radial(AR) y la arteria colateral radial (ACR) (Fig. 2). El pedículo principal, en todos los casos, es una rama de la ARR (arteria del Extensor Carpi Radialis Longus) que da tras la salida del pedículo vascular para el músculo Braquiorradialis y entra en el músculo aproximadamente $30 \mathrm{~mm}$ de su origen subdividiéndose en 4 o 5 ramas musculares que vascularizan prácticamente todo el músculo. Presenta un trayecto primero transversal y luego ascendente hacia el codo paralelo al nervio radial, cruzándolo anteriormente a nivel de su bifurcación en rama sensitiva y rama motora interósea posterior, y dividiéndose a este nivel en las ramas musculares de tal forma que rodean anterior y posteriormente al nervio, haciendo imposible hacer libre el colgajo muscular sin sacrificar el nervio radial, hallazgo constante en 28 de las 29 disecciones (Fig. 3).

En 11 disecciones (37.9\%) ésta es la única fuente vascular del ECRL, constituyendo un único pedículo vascular dominante, con un patrón vascular tipo I. En 18 disecciones $(62,1 \%)$ existe un pedículo menor proveniente de la AR que vasculariza la porción distal del músculo, lo que supone aproximadamente un $20 \%$ del mismo, desde 6-7 cm de su inserción en la cresta supracondílea hasta la unión musculotendinosa; en estos casos el patrón vascular corresponde a un tipo II, con un pedículo dominante y uno menor, siendo por tanto el patrón más frecuentemente encontrado. La ACR, rama de la arteria braquial profunda, se anastomosa proximalmente con la ARR, dando 1 o 2 ramas musculares que vascularizan el polo superior y

Tabla I: Calibres y Longitud de pedículos de los músculos ECRL y ECRB (distancias en mm)

\begin{tabular}{lcccc}
\hline & \multirow{2}{*}{ ECRL } & \multicolumn{2}{c}{ ECRB } & Longitud de pedículo \\
Media & Calibre & Longitud de pedículo & Calibre & 27,77 \\
Desviación Estándar & 1,73 & 32,32 & 1,11 & 8,59 \\
Rango & 0,40 & 7,96 & 0,28 & $15-44$ \\
\hline
\end{tabular}




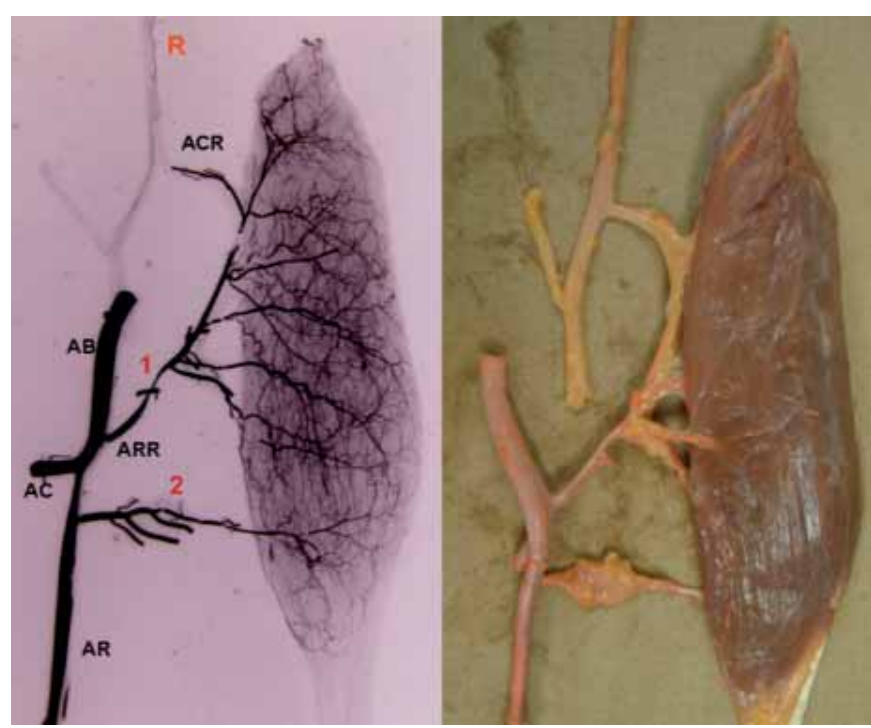

Fig. 2. Vascularización del ECRL, correlación de hallazgos anatómicos y radiológicos. El pedículo principal (1) sale en todos los casos de la ARR y el pedículo menor de la AR .La ACR entra en el músculo con una rama motora de nervio radial y se anastomosa con ramas del pedículo principal. La ARR es rama de la AR en $86,20 \%$ de las disecciones, de la $A B$ en $10,34 \%$ y de la $A C$ en $3,34 \%$. (AB: arteria braquial; AC: arteria cubital; AR: arteria radial; ARR: arteria recurrente radial; ACR: arteria colateral radial; R: nervio radial).
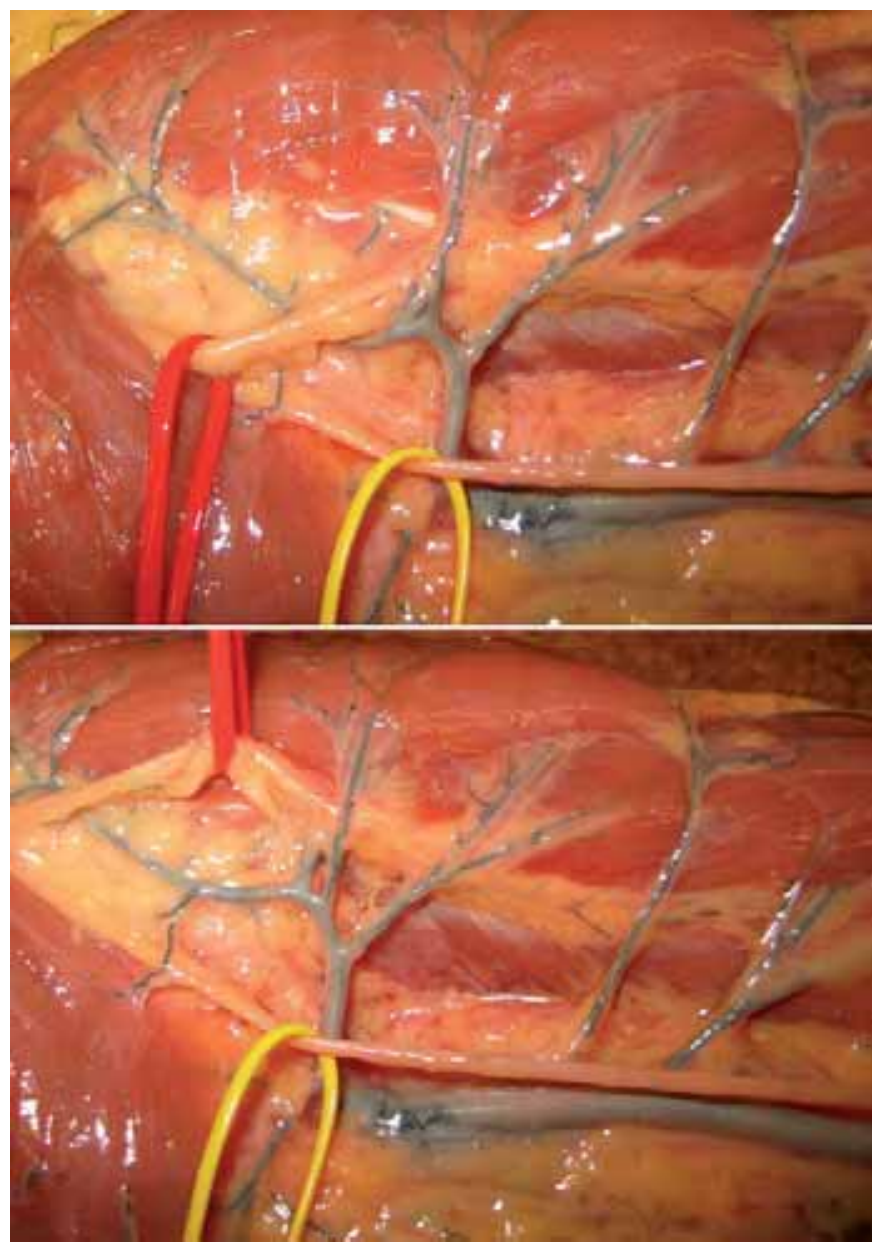

Fig. 3. Relación del pedículo vascular del ECRL y el Nervio Radial. La rama motora del nervio radial (rojo) atraviesa las ramas musculares del pedículo principal del ECRL pasando anteriormente y luego posteriormente. La rama sensitiva del nervio radial (amarillo) pasa para- la inserción del músculo, hallazgo consistente en todos los cadáveres examinados.

\section{Extensor Carpi Radialis Brevis}

El ECRB es irrigado por dos fuentes arteriales cuyo origen son la ARR y la AR. El pedículo principal, que denominamos arteria del Extensor Carpi Radialis Brevis, provino en 9 brazos $(31 \%$ de las disecciones) de la ARR y en 20 brazos $(69 \%$ de las disecciones) de la AR, irrigando el $75 \%$ del músculo aproximadamente $7 \mathrm{~cm}$ proximalmente a la unión musculotendinosa. Presenta un trayecto descendente y paralelo a la rama sensitiva del nervio radial y anterior a ésta.

Según los hallazgos encontramos 3 tipos de patrones vasculares en el ECRB:

-Tipo I (6 brazos, 20,68\% de los casos): un único pedículo dominante cuyo origen está en la ARR.

-Tipo II ( 23 brazos, 79,31\% de los casos): un pedículo dominante y uno o varios pedículos menores. En 20 casos $(68,96 \%$ de las disecciones) el pedículo dominante sale de la AR y los menores, uno proximal rama de la ARR que proporciona la vascularización del polo superior de músculo a nivel de la inserción muscular en el epicóndilo y otro distal, rama de la $\mathrm{AR}$, que vasculariza la porción muscular distal inmediatamente adyacente a la unión musculotendinosa, constituyendo el patrón que denominamos IIA. En 3 casos (10,34\% de las disecciones) el pedículo dominante sale de la ARR (como en el patrón I) y presenta un pedículo menor distal rama de la AR para la unión musculotendinosa; denominamos a este patrón vascular IIB.

\section{Discusión}

\section{Comparación de los resultados con la literatura}

No hemos encontrado contradicciones aunque sí ciertas diferencias entre los hallazgos de nuestro trabajo y los datos reflejados en la literatura (6-13) (Tabla II). La mayor parte de los trabajos publicados atribuyen a la ARR la mayor parte de la vascularización del ECRL y ECRB y de manera ocasional, atribuyen un flujo menor a otras arterias como la AR y ACR; habitualmente son trabajos que estudian de manera global el antebrazo y someramente estos músculos. Se atribuye en dos publicaciones a ambos músculos un patrón vascular de tipo I y los pedículos dominantes se observan como ramas de la ARR.

En nuestro trabajo hemos encontrado la existencia de 2 tipos de patrones según la clasificación de Mathes y Nahai, el tipo I y tipo II para ambos músculos. A diferencia de trabajos previos, nuestro estudio refleja que el patrón tipo II es el más frecuente tanto 
Tabla II: Referencias históricas del estudio de la vascularización de los músculos ECRL y ECRB

Dubreuil-Chambardel (6), 1926 Describe la vascularización del ECRL, ECRB y Braquiorradialis y observa una rama arterial muscular para cada músculo proveniente de la ARR.

Salmon (7), 1933

Zbrodowsky et al (12), 1982

Parry et al (8,9), 1988 y 1989

Revol et al (10), 1991

Yoshiharu (11) et al, 1996

Schneberger (13) et al , 2001
Denomina arteria muscular del Extensor Carpi Radialis Longus a la arteria principal del ECRL que proviene de la ARR y observa la existencia de una duplicatura de esta arteria principal en el $25 \%$ de los casos. Describe una vascularización accesoria para el ECRL proveniente de pequeñas arterias ramas de la ARR, AR y de la ABP. El ECRB presenta dos arterias principales, una procedente de la ARR y otra de la AR.

Estudio de la vascularización de los tendones del ECRL y ECRL a nivel del carpo observando dos fuentes vasculares principales: la rama dorsal del carpo proveniente de la AR y la AIA.

Estudio de la anatomía vascular de los músculos de la extremidad superior. Describen la ARR como la arteria que perfunde mayoritariamente los músculos del compartimento lateral del antebrazo; establecen en porcentajes la perfusión total del ECRL, determinando que el $80 \%$ proviene de la ARR, el 10\% de la AR y el $10 \%$ de la AC y clasifican al ECRL como músculo Tipo 1 según la clasificación de Mathes y Nahai

Estudio de la anatomía vascular de los músculos del antebrazo: observan unas 12 ramas arteriales musculares de media para el ECRL provenientes de la ARR y unas 9 de media para el ECRB provenientes de la ARR y de la AR.

Describen los angiosomas del antebrazo: observan que ambos músculos son de tipo 1, vascularizados proximalmente de manera constante por la ARR y ocasionalmente por la AB y la ABP y distalmente por la AR.

Estudian la vascularización de la porción proximal del ECRB, observando que la ARR y la ACR proporcionan la mayor parte de las ramas musculares y la AIR una mínima contribución vascular.

ECRL:extensor carpi radialis longus; ECRB: extensor carpi radialis brevis; AR: arteria radial; ARR:arteria recurrente radial; AIA: arteria interósea anterior; ABP: arteria braquial profunda;AB: arteria braquial; AC: arteria cubital; ACR: arteria colateral radial; AIR: arteria interósea recurrente

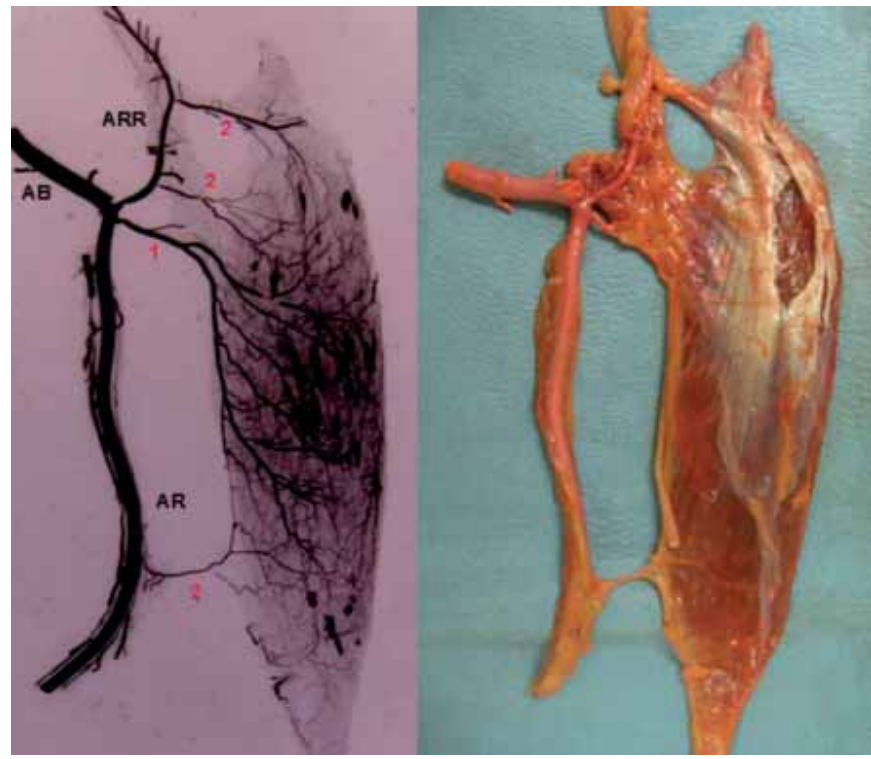

Fig. 4. Vascularización del ECRB, correlación de hallazgos anatómicos y radiológicos. El pedículo principal (1) sale en el $68,96 \%$ de los casos de la AR y existen pedículos menores (2) en el $79,31 \%$ de las disecciones provenientes de la ARR y de la AR. (AR: arteria radial, ARR: arteria recurrente radial).

en el ECRL como en el ECRB, apareciendo en el $62 \%$ y $79,3 \%$ de los casos respectivamente. Asimismo encontramos que el pedículo dominante del ECRB, que denominamos arteria del ECRB, sale en el 68'9\% de los casos de la AR. Esto es coherente con las obser-
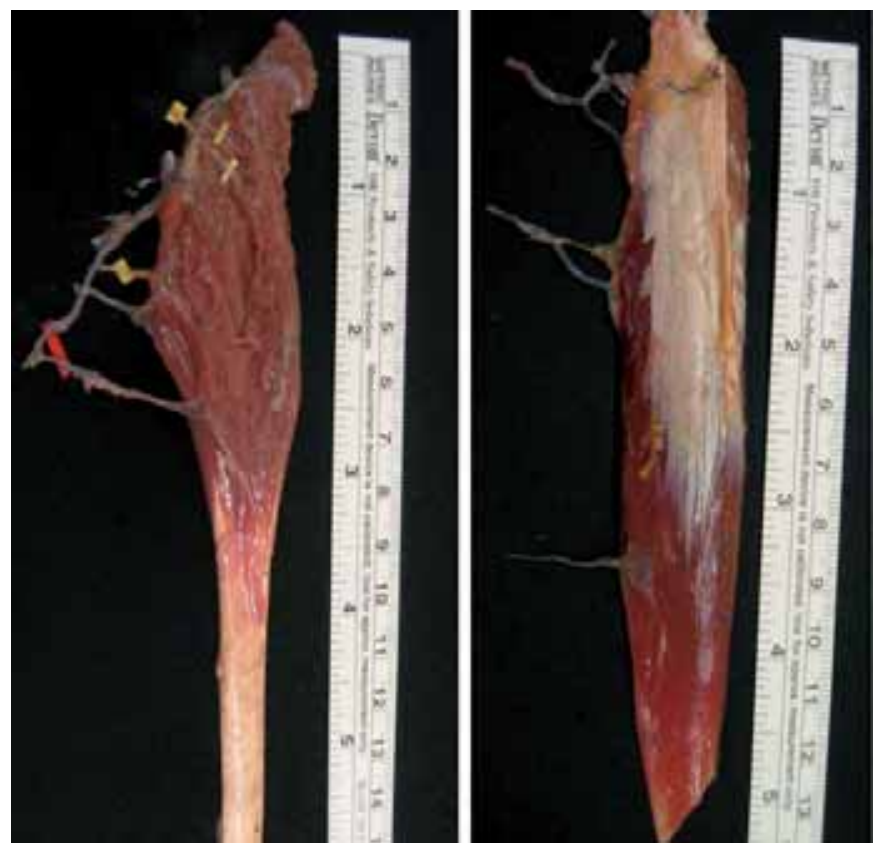

Fig. 5. Longitud y contenido fascial del ECRL (izquierda) y ECRB (derecha).

vaciones de Salmon (7) que encontró dos pedículos principales para el ECRB, uno de la ARR y otro de la AR (lo que sería un patrón vascular tipo III); nosotros consideramos que el pedículo saliente de la AR es habitualmente de mayor calibre y está en posición 
más central en el músculo que el saliente de la ARR (habitualmente rama distal del pedículo del ECRL) por lo que sería el pedículo principal.

\section{Aplicabilidad Clínica de los Colgajos Musculares de ECRL y ECRB}

Los músculos ECRL y ECRB junto con el Extensor Carpi Ulnaris (ECU) son músculos sinérgicos que extienden la muñeca. El uso clínico en Cirugía Reparadora del ECRL y ECRB ha sido limitado fundamentalmente a transferencias tendinosas para parálisis de nervios mediano o cubital $(1,2)$. Asimismo el ECRL ha sido utilizado de manera puntual como colgajo muscular pediculado por Ohtsuka (14) y por Janevicius (15) para la cobertura de defectos de pequeño tamaño alrededor del codo.

Según Doi (16), para la selección de un músculo donante en una transferencia muscular funcional, éste debe ser parecido en forma, tamaño, longitud de fibras y área de corte transversal fisiológica al músculo que se busca sustituir. En reanimación facial el objetivo fundamental es sustituir el músculo principal de la sonrisa, el cigomático mayor. En este sentido estos músculos presentan unas características anatómicas que hacen que sean potencialmente idóneos para su utilización en reanimación facial como colgajos libres funcionales (Fig. 5). Ambos presentan una longitud de las fibras musculares de entre $7-10 \mathrm{~cm}$, lo que es similar a la longitud del cigomático mayor y supone una ventaja en reanimación facial, ya que el poder transferir un músculo sin la división de sus fibras permite una mayor contracción muscular; además al ser músculos finos, producirán un contorno adecuado en la cara. Ambos presentan un gran contenido facial, fundamentalmente el ECRB, lo que permite un adecuado anclaje de las suturas para realizar los vectores de tracción de la sonrisa. Presentan una inervación motora del nervio radial habitualmente proximal a su división en rama sensitiva y rama interósea posterior (17).

En 1995 Manktelow (3) presentó una serie clínica de 8 pacientes en los que usó el ECRB como colgajo muscular libre para reanimación facial, con éxito clínico en 7 de los 8 casos. No obstante, reflejó problemas fundamentalmente en la cicatriz de la zona dadora y 2 pacientes presentaron disminución de fuerza en la muñeca con ejercicios de fuerza intensos. Se recomienda su uso en pacientes mayores, en los que existe una mayor laxitud de tejidos y la fascia del ECRB permite un excelente punto de anclaje.

En nuestro estudio observamos que la vascularización de ambos músculos se produce a expensas de un pedículo dominante (patrón vascular tipo I ) o un pedículo dominante y varios menores(patrón vascular tipo II) con la existencia de abundantes conexiones vasculares entre las fuentes arteriales que hace suponer que la totalidad de los músculos podrían ser transferidos por su pedículo principal. En 28 casos $(96,5 \%$ de las disecciones) hemos encontrado que el nervio radial atraviesa anterior y posteriormente varias ramas arteriales musculares del pedículo principal del ECRL, con lo que para poder hacerlo libre habría que sacrificar la rama motora del nervio radial, una secuela que no es asumible al existir otras opciones reconstructivas.

Ambos músculos presentan una longitud de pedículo corta y un calibre pequeño $(1,73+$ +DS 0,4 el ECRL y $1,11+-$ DS 0,28 el ECRB) aunque suficiente para la realización con garantías de una anastomosis microvascular; este calibre puede ser aumentado si se aumenta la disección hasta la ARR, cuando el pedículo principal es rama de éste, donde el calibre medio es de 1,94 +-DS 0,22.

\section{Conclusiones}

Los músculos ECRL y ECRB presentan dos tipos de patrones vasculares: tipo I y tipo II, siendo más frecuente en nuestro trabajo el tipo II, que hacen que ambos puedan ser transferidos como colgajos libres por su pedículo principal.

Ambos músculos presentan un tamaño, contorno, contenido fascial importante para el anclaje de suturas, así como una longitud de pedículo y calibre vascular adecuados para su transferencia microvascular libre en reanimación facial.

De los dos, el más realizable como colgajo libre es el ECRB, ya que la relación anatómica del nervio radial con el pedículo del ECRL hace que no sea posible su transferencia como colgajo libre sin sacrificar la rama motora del nervio radial en la mayor parte de los casos.

\section{Dirección del autor}

Dr. Andrés Rodríguez Lorenzo

Avda. Augusto García Sánchez, 14-12 B.

36001 Pontevedra, España.

e-mail: a.rodriguez.ps@gmail.com

\section{Bibliografía}

1. Burkhalter WE.: "Median nerve palsy". En: Green DP, ed. Operative hand surgery. $2^{\text {nd }}$ ed.New York : Churchill Livingstone, 1988, Pp:1499-1534

2. Omer GE.: "Ulnar nerve palsy". En : Green Dp, ed. Operative hand surgery. $2^{\text {nd }}$ ed. New York : Churchill Livingstone, 1988, Pp:15351554 
3. Manktelow RT, Binhammer P.: "Clinical assessment of the extensor carpi radialis brevis muscle for reconstruction of the smile". Proceedings of the $4^{\text {th }}$ International Muscle Symposium, March $23^{\text {rd }}-25^{\text {th }}, 1995$, Zurich. M. Frey, P. Giovanoli(Eds.)

4. Mathes,S.J., and Nahai, F.: "Reconstructive Surgery: Principles, Anatomy and Technique", $1^{\text {st }}$ Ed. New York: Churchill-Livingtone \& Quality Medical Publishing, 1997, Pp. 38-45.

5. Tang M, Geddes CR, Yang D, Morris SF.: "Modified Lead Oxidegelatin Injection Technique for vascular studies". Journal of Clinical Anatomy 2002. 1:73.

6. Dubreuil-Chambardel, L.: "Traite des Variations du Systeme Arteriel:Variations des Arteries du Membre Superieur". Paris:Masson,1926.

7. Salmon, M.: "Arteries of the Muscles of the Upper Extremity". In G.I. Taylor and R.M. Razaboni (Eds.), Arteries of the Muscles of the Extremities and the Trunk. St. Louis: Quality Medical Publishing, 1994, Pp: 87-97.

8. Parry AW, Ward JW, Mathes SJ.: "Vascular anatomy of the upper extremity muscles" .Plastic and Reconstructive Surgery, 1988, 81(3): 358.

9. Parry W, Mathes SJ.: "Blood supply of the upper extremity muscles related to functional tendon transfers". Clinics in Plastic Surgery, 1989,16(3):531.
10. Revol MP, Lantieri L, Loy S, Guerin-Surville H.: "Vascular anatomy of the forearm muscles: a study of 50 dissections". Plastic and Reconstructive Surgery, 1991, 88(6):1026.

11. Yoshiharu I, Taylor I.: "The angiosomes of the forearm:anatomic study and clinical implications". Plastic and Reconstructive Surgery., 1996,98(2):195.

12. Zbrodowsky A, Gajisin S, Grodecki J.: "Vascularization of the tendons of the extensor pollicis longus, extensor carpi radialis longus and extensor carpi radialis brevis muscles" .Journal Anatomy, 1982,135(2):235.

13. Scheneeberger AG, Masquelet AC.: "Arterial vascularization of the proximal extensor carpi radialis brevis tendon". Clinical Orthopaedics and Related Research, 2002, 398: 239.

14. Ohtsuka H, Imagawa S.: "Reconstruction of a posterior defect of the elbow joint using an extensor carpi radialis longus myocutaneous flap:case report". British Journal of Plastic Surgery, 1985, 38: 238.

15. Janevicius RV, Greager JA.: "The extensor carpi radialis longus muscle flap for anterior elbow coverage". The Journal of Hand Surgery, 1992,17A(1):102.

16. Doi K, Hattori.: "Basic science behind functioning free muscle transplantation". Clinics in Plastic Surgery,2002, 29:483.

17. Abrams RA., Ziets RJ, Lieber RL, Botte MJ.: "Anatomy of the radial nerve motor branches in the forearm". The Journal of Hand Surgery, 1997, 22A(2): 232. 